\title{
Assessing the spatial accessibility of hospital care in Sichuan Province, China
}

\author{
Jay Pan, ${ }^{1,2}$ Huiran Liu, ${ }^{1}$ Xiuli Wang, ${ }^{3}$ Hongmei Xie, ${ }^{1}$ Paul L. Delamater ${ }^{4}$ \\ ${ }^{1}$ West China School of Public Health, Sichuan University, Chengdu; ${ }^{2}$ West China Research \\ Center for Rural Health Development, Sichuan University, Chengdu; ${ }^{3}$ College of \\ Architecture and Environment, Sichuan University, Chengdu, China; ${ }^{4}$ Department of \\ Geography and Geolnformation Science, George Mason University, Fairfax, VA, USA
}

\begin{abstract}
Regional disparities in geographical access to hospital care are found throughout China. Understanding variations in the spatial accessibility of hospital care has the potential to provide decision support in healthcare planning. This study examines the hospital system in the Sichuan Province in China, which provides healthcare for more than 80 million people. We examine the impacts of accessibility characterisation via the conventional measurement approach by comparing the results to those derived using a floating catchment area approach. Employing a geographical information system based on pop-
\end{abstract}

Correspondence: Jay Pan, West China School of Public Health, Sichuan University, Ren Min Nan Road 17, Sichuan, Chengdu 610041, China.

Tel: +86.28 .8550 .1096 - Fax: +86.28 .8550 .1528 .

E-mail: panjie.jay@scu.edu.cn

Key words: Spatial accessibility; Hospital care; Enhanced two-step floating catchment area method; China.

Conflict of interest: the authors declare no potential conflict of interest.

Contributions: JP, HL, data collecting; JP, XW, data analysing; JP, HL, HX, PD, manuscript writing; PD, manuscript reviewing and reference search.

Acknowledgements: we would like to thank the National Natural Science Foundation of China (Grant No. 71303165), the China Postdoctoral Science Foundation (Grant No. 2013M540706 and 2014T70863), Sichuan University (Grant No. skqx201401 and 2015SCU04A19), Health and Family Planning Commission of Sichuan Province (Grant No.150112), and China Medical Board (Grant No. 13-167) for their financial support. The authors are responsible for all remaining errors.

Received for publication: 12 June 2015.

Revision received: 9 September 2015.

Accepted for publication: 22 October 2015.

(C) Copyright J. Pan et al., 2015

Licensee PAGEPress, Italy

Geospatial Health 2015; 10:384

doi:10.4081/gh.2015.384

This article is distributed under the terms of the Creative Commons Attribution Noncommercial License (by-nc 3.0) which permits any noncommercial use, distribution, and reproduction in any medium, provided the original author(s) and source are credited. ulation and hospital administrative data, we conducted a provincewide study of the spatial accessibility of hospital care in Sichuan Province, China. A shortest-path analysis and the enhanced two-step floating catchment area (E2SFCA) method were implemented. Substantial differences between these two approaches were found, including a roughly $15 \%$ difference in the total number of under-served areas. Generally, spatial accessibility was higher in the eastern regions of Sichuan. More than 5.5 million people were found to have limited access, with large variations across the province. These results indicate that the official method used by policy makers in China may not capture the true nature of spatial accessibility throughout the region. We recommend that the E2SFCA method be implemented for health services research in China, providing decision makers with more accurate information when setting healthcare policies.

\section{Introduction}

Access to healthcare depends on characteristics of both the delivery system and the population in need of such services (Aday and Andersen, 1974). However, the assessment of overall access to healthcare has proven to be difficult due to the multifaceted nature of the concept and the complex set of interactions occurring between populations and services. Often, a distinction is made between potential and realised access. Specifically, while the former is based solely on the ability of the system to provide services for the population, realised access depends on actual utilisation of these services (Aday and Andersen, 1974; Joseph and Phillips, 1984).

Penchansky and Thomas (1981) defined access as a measure of fit between the population and the services, identifying five distinct dimensions of potential access. These dimensions can be classified into spatial components (accessibility and availability) and non-spatial components (affordability, accommodation and acceptability) (Khan, 1992). Accessibility describes the burden of travel between locations, while availability describes the number of services available. As these two entities are commonly not independent of each other, we are often forced to deal with services falling within a specific distance. This fusion of availability and accessibility has been referred to as the spatial accessibility of healthcare services (Guagliardo, 2004).

Spatial accessibility is recognised as an important component in the evaluation of a population's overall access to healthcare (World Health Organization, 2000; Horev et al., 2004; Berke and Shi, 2009; Andersen et al., 2011). Disparities in spatial accessibility result from the geographical configuration of delivery locations, population distribution and the transportation infrastructure linking them together. 
Specifically, healthcare services are offered from a fixed set of locations but are required to serve a population considered to be continuously distributed across a landscape (Joseph and Phillips, 1984). Although disparities in spatial accessibility are inevitable due to this configuration, some populations are more adversely affected than others. For example, rural populations are often required to travel much longer distances to access services and are likely to have fewer services available (Laditka et al., 2009; Ranga and Panda, 2014).

In China, there are large disparities in the spatial accessibility of healthcare recourses among regions. In the 2010 national survey of rural impoverished counties (representing $36.1 \%$ of total rural residents in China, 242.39 million people), $34.6 \%$ of the respondents, who reported not having timely access to medical care, attributed distance to a medical institution as the barrier (Department of National Bureau of Statistics of China, 2012). For urban areas, including suburban areas, the problem also exists, although the magnitude appears smaller. In 2010, 1.4\% of urban residents who chose not to be hospitalised (against the advice by a physician) reported too far away from the hospital as the reason (State Council, 2010). However, the severity of this problem differs among Chinese cities, varying from 0 to $10.7 \%$ and indicating that the spatial accessibility of hospital care is an issue of concern in some urban regions (summarised from State Council, 2010).

In an effort to alleviate disparities in healthcare access, an undertaking under the slogan promoting the progressive equalization of the basic public health services was introduced as one of the five key reform projects of the Chinese healthcare reform of 2009 (Communist Party of China, 2009). Table 1 summarises related policies introduced during 2009-2012 by China's central Government. More than 2116 county hospitals and 8859 primary health centres were planned for construction or given additional support during this period. By 2011, investment at the level of 43 billion Yuan (about USD 6.7 billion) had been made for the support of hospital construction and 13 billion Yuan (about USD 2 billion) for medical equipment (Development and Reform Commission of China, 2012).

Given this large investment with respect to healthcare services, a challenge of where to locate the new facilities emerges. Because the distribution of new facilities has the potential to affect healthcare access for a large number of people for a long time to come, both equity and efficiency should be carefully considered. From a regional health planning perspective, correctly identifying under-served areas must be an essential component of this process. Currently, policy makers use a rudimentary, regional availability approach (ratio of services per population within administrative boundaries) to characterise the spatial accessibility of healthcare services. An advantage of this approach is that it is easy to calculate and interpret what is needed. Implementation is generally straightforward as data for healthcare resources (such as physicians, hospital beds), population counts and the regional administrative boundaries are well known and understood by both policy makers and the general population (Florin et al., 1994; Luo and Qi, 2009). However, the assumptions regarding the regional availability approach do not accurately reflect how access is experienced in real world settings (Wing and Reynolds, 1988). To address this issue, a number of spatial techniques have been developed based on the gravity model (e.g., Joseph and Bantock, 1982) and implemented in a geographical information system (GIS). Specifically, the floating catchment area (FCA) family of spatial accessibility metrics (Luo and Qi, 2009) provides an alternative, GIS-based approach to characterise the spatial accessibility of healthcare resources.

GIS-based spatial accessibility models have rarely been applied for healthcare issues in China; indeed, they have yet to be incorporated by Chinese policy makers or health policy researchers. To illustrate their potential advantages, this study employed the enhanced two-step floating catchment area (E2SFCA) method to analyse the spatial accessibility of hospital services in the Sichuan Province. There are two main reasons for choosing an entire province as the study unit. First, most of the public policies regarding financial support for healthcare are made at the provincial level in China; second, the results may potentially provide support for future policymaking efforts and healthcare resource allocation in Sichuan Province.

We selected Sichuan Province as the study area in an effort to strengthen the possibilities to generalise the results. Sichuan is one of the largest provinces in China, both with regard to area and to population. The level of economic development and availability of healthcare resources, as well as population are unevenly distributed throughout the province. Further, Sichuan has a variable topography, which affects travel patterns, that is similar throughout China as a whole. The first contribution of this study would be to apply GIS-based methods, the shortest path analysis (Kwan, 1998) and the E2SFCA method (Luo and $\mathrm{Qi}, 2009$ ), to a healthcare system covering a large area and a big population, for which these methods have rarely been applied. The second contribution would be to compare the effectiveness of the conventional method with the GIS-based methods in identifying the under-served areas for hospital care. From a public policy perspective, our results

Table 1. Policy summary for China's healthcare reform 2009-2012.

Year Contents summary

2009 Support the construction of 986 county-level hospitals (including traditional Chinese medicine hospitals), 3549 township hospitals, and 1154 urban community health service centres and other basic medical and health institutions in the whole country

2010 Based on 2009, add around 830 county-level hospitals (including traditional Chinese medicine hospitals), about 1900 township hospitals, about 1250 urban community health service centres, and more than 8000 village clinics

2011 Complete the task of constructing the rural three-level, health service network and the urban community health service organisation, and add more than 300 county-level hospitals (including traditional Chinese medicine hospitals), more than 1000 township hospitals, and more than 13,000 village clinics to support the construction on the basis of the previous two years, so that each county has at least 1 county-level hospitals reaching the level 2 (class A)* hospital and 1 to 3 reaching the standard township hospitals, each administrative village has a clinic, and each street has a community health service institution. In addition, allocate mobile touring medical service cars to the Midwestern remote and mountainous areas

2012 According to the principle of filling up the gaps, continue to increase support for the standardised construction of township hospitals

*Generally, hospitals in China are designated as a primary (level 1), secondary (level 2) or tertiary (level 3) hospital by China's Ministry of Health, since 2013 named the Health and Family Planning Commission. For each level, there are three sub-levels: class A, B, and C. The key criterion for the classification is the number of hospital beds at the facility. Hospitals having many beds are generally given a higher classification level. 
should provide important insights that have the potential of influencing future efforts to identify areas with healthcare shortage and to develop strategies that could improve population-level access.

\section{Materials and Methods}

\section{Study area and data}

Sichuan, located in the Southwest, roughly between latitudes $92^{\circ} 21^{\prime} \sim 108^{\circ} 12^{\prime} \mathrm{E}$ and longitudes $26^{\circ} 03^{\prime} \sim 34^{\circ} 19^{\prime} \mathrm{N}$, is the fifth largest province $\left(486,000 \mathrm{~km}^{2}\right)$ of China. It is an important industrial centre province due to its agricultural and mineral resources. There are 181 counties in Sichuan, whose total population was 80.76 million in 2012 (National Bureau of Statistics of China, 2013).

Hospital data were acquired from the provincial Health Bureau. Three indicators were used to characterise hospital capacity: the number of hospital beds, the number of staff, and the number of medical doctors. The staff includes registered doctors, assistant doctors, registered nurses, pharmacists and laboratory technicians. The latitude and longitude of hospital locations were geocoded using the hospital names and addresses.

We used the road network to compute the shortest travel times from the residents' locations to hospitals. The road network and administrative boundaries data were gathered from the 1:250,000-scale Topographic Database of the National Fundamental Geographic Information System of China provided by National Geomatics Center of China. According to the Chinese road technical standards, travel speeds are set according to the quality of the road, i.e. as 100, 80, 60, 40 and $20 \mathrm{~km} / \mathrm{h}$ for highways, state roads, provincial roads, county roads and village roads, respectively.

The population counts of the administrative zones (counties) were

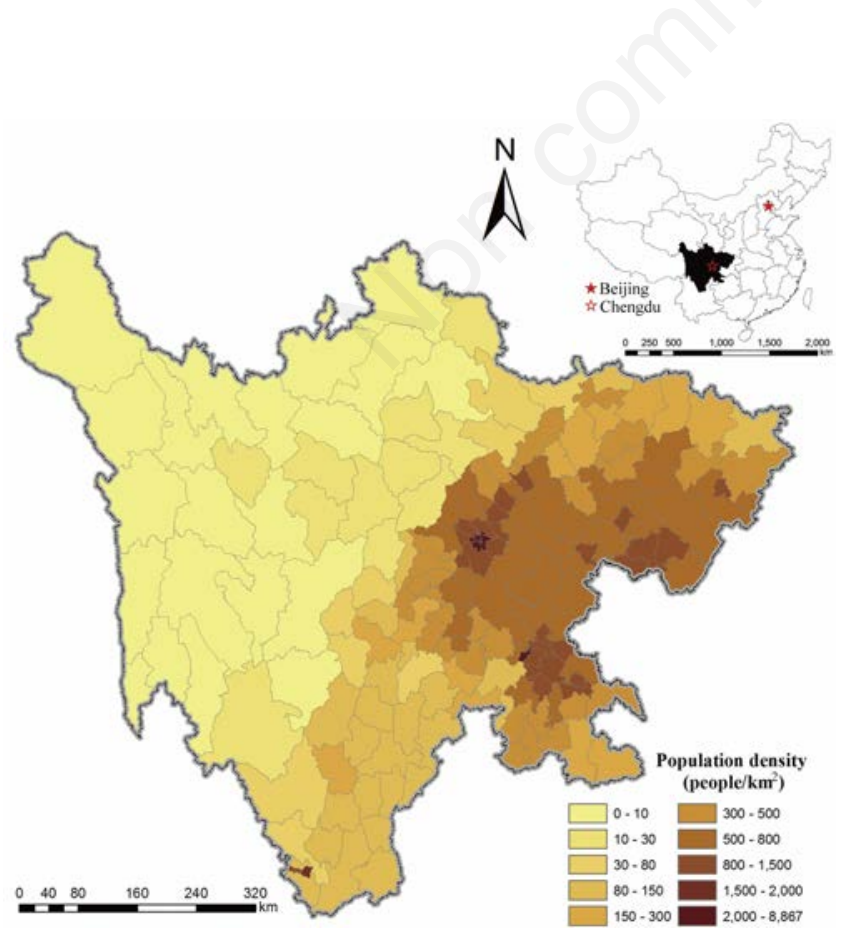

Figure 1. Position and population density per county in Sichuan Province, China. obtained from Sichuan Province Statistical Yearbook 2013, published by the Department of the Statistics Bureau of Sichuan. The population data were disaggregated using a 60 arc-second $(\sim 2 \mathrm{~km})$ grid allocating the total population of each county evenly over the $\sim 4 \mathrm{~km}^{2}$ units provided by this grid. This method was chosen because a higher resolution of population data was not available for the study area.

Figure 1 shows Sichuan's population density by county, which is relatively high in the East and declinines generally and in the western direction within the province. Qingyang District, one of the districts within Chengdu City (the capital of Sichuan), has the highest population density ( 8867 residents per $\mathrm{km}^{2}$ ), while Daocheng County, located in the Southwest has the lowest (4.58 residents per $\mathrm{km}^{2}$ ). Although the hospital distribution appears relatively consistent with the population, the 1547 hospitals are unevenly distributed throughout the province (Figure 2). Figure 3 displays the road network, which is denser in the South and East.

\section{Statistical approach}

The conventional method to define the spatial accessibility is simply to use population ratios with respect to the health services that fall within predefined, administrative boundaries. However, there are two main assumptions that should cause concern as they are generally not valid in real-world care-seeking scenarios. (Joseph and Phillips, 1984; Luo and Qi, 2009): i) residents within the administrative region all have equal access to health resources within the region; ii) residents of a region do not travel beyond the boundaries of their region to seek care elsewhere (Wing and Reynolds, 1988). Employing different levels of data aggregation (resolution) can strengthen the validity of one of these two assumptions but not both at the same time. For example, using more disaggregated data (smaller areal units) will make the first assumption more realistic but would also decrease the validity of the

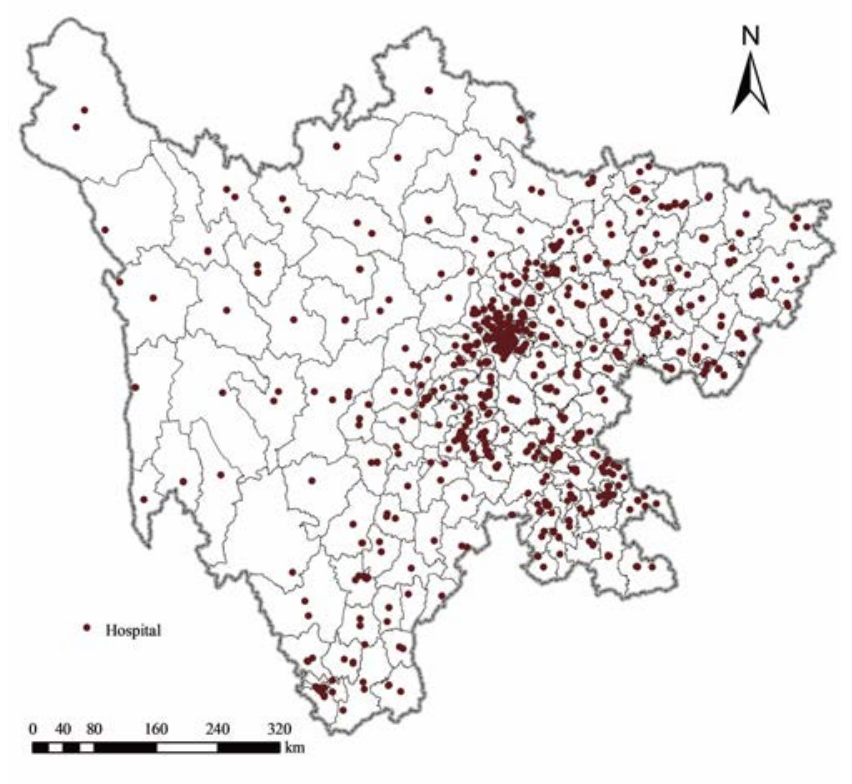

Figure 2. Hospital distribution as of 2012 in Sichuan Province, China. 
second one (Kivell et al., 1990). The inverse is true if more aggregated data are used (Makuc et al., 1991; Luo and Qi, 2009).

A gravity-based model estimates spatial accessibility by incorporating supply, demand and distance concurrently (Weibull, 1976; Joseph and Bantock, 1982). It takes the general form:

$$
A_{i}^{G}=\sum_{j=1}^{n} \frac{S_{j} f\left(d_{i j}\right)}{\sum_{k=1}^{m} P_{k} f\left(d_{k j}\right)}
$$

where $A_{i}{ }^{G}$ denotes the spatial accessibility at location $i$, and $n$ and $m$ the sets of all medical institutions and population locations, respectively. $S_{j}$ represents the supply at location $j(e . g$, the capacity of medical institution, such as the number of hospital beds), while $f(d)$ is a geographic impedance function, based on $d_{i j}$, the travel time or distance between $i$ and $j$, and $P_{k}$ represents the potential demand at location $\mathrm{k}(e . g$., the number of people). The basic gravity model assumes the spatial accessibility of health services for a population is equal to the sum of impedance-weighted supply-to-demand ratios of all nearby medical sites. The advantages of the gravity model are as follows: first, $A_{i}{ }^{G}$ accounts for all the three determinants of spatial accessibility (supply, demand and distance) within a single model (Luo and Qi, 2009); second, the two assumptions of the population ratio approach are not required. The main weakness of the gravity model is that the output unit of $A_{i}{ }^{G}$ is not easily interpretable and that limits its use in healthcare planning or resource evaluation efforts.

FCA metrics represents a special form of the gravity model, integrating the theoretical advantages of the model with an easily understood output unit (supply per person). The first implementation was the twostep floating catchment area method (2SFCA) (Radke and Mu, 2000; Luo and Wang, 2003), which is similar to the regional availability method, but allows the boundaries to float based on a user-specified distance from each facility and population location. The 2SFCA does not consider distance decay within catchments (i.e., all locations inside of the catchment are assumed to have equivalent access). Luo and Qi (2009) address this issue by applying the E2SFCA metric, in which each catchment area is divided into multiple sub-zones of different weight values based upon a distance decay function $f(d)$, which can be adjusted depending on the travel behaviour associated with the service. The exponential function $\left(f(d)=e^{-\beta d}\right)$, the inverse-power function $\left(f(d)=e^{-\beta}\right)$, and the Gaussian function $\left(f(d)=\exp \left(-d_{i j}{ }^{2} \beta\right)\right)$ are often used forms of $f(d)$, where $\beta$ is the impedance coefficient indicating the extent to which the travel cost (distance or travel time) would impact on various individuals' access to the services in question (Kwan, 1998).

Several other methods, such as the three-step floating catchment area method (3SFCA) and modified two-step floating catchment area method (M2SFCA), have been proposed as extensions or modifications of the E2SFCA. However, there is no consensus that any method is superior (McGrail, 2012; Wan et al., 2012; Delamater, 2013). Considering that the E2SFCA is the most widely used in recent related studies and recommended as the standard floating catchment area method (Langford et al., 2012; Dewulf et al., 2013), this metric was employed in our study.

A 60-min driving zone was defined as the catchment area for hospitals (McGrail and Humphreys, 2009). In this respect, it must be noted that the physical terrain in Sichuan is complex, with mountains, plateaus and hills occupying about $97.46 \%$ of the total land area. A majority of Sichuan's population (75.78\%) is considered rural. Previous literature suggests that the catchment size for rural areas should extend to a larger value, such as 60 minutes, in an effort to incorporate isolated areas.

Each catchment was divided into subzones (0-10, 10-30 and 30-60 minutes). In the Chinese context, 10 minutes is viewed as initial impedance that presents as small barrier to accessing hospital care, while 30 minutes is viewed as standard travel time advocated by various health plans. Given the considerations, 10 and 30 minutes were used as the cut-off values to define the ranges of the catchment zone. Weights $(W r)$ for each subzone were calculated using the following Gaussian function as of the three common forms of $f(d)$, the Gaussian function is considered the most appropriate for simulating the distance decay effect (Kwan, 1998; Wang, 2007; Cromley and McLafferty, 2011):

$$
w_{r}=f\left(d_{i j}\right)=\exp \left(-d_{i j}^{2} / \beta\right)
$$

The Gaussian weight function is highly dependent upon the impedance coefficient, $\beta$. Large values of $\beta$ (the impedance coefficient) are used when people are less willing to travel long distances to access care (Luo and Wang, 2003). The mean travel time for each subzone $(5,20$, and 45 minutes) were used as $d_{i j}$ values in the calculation. As suggested by Wan et al. (2012), 0.01 is a critical point for Gaussian function approaching zero. Using the midpoint of each travel subzone, a $\beta$ value of 440 provides $W_{r}$ values of $0.945,0.403$ and 0.01 for the $0-10,10-30$ and 30-60 minute subzones, respectively

The first step of the E2SFCA is to calculate the supply to demand ratio for each hospital $\left(W R_{j}\right)$ :

$$
W R_{j}=\frac{S_{j}}{\sum_{k \in\left\{d_{k} \in D_{r}\right\}} P_{k} W_{r}}=\frac{S_{j}}{\sum_{k \in\left\{d_{k} \in D_{1}\right\}} P_{k} W_{1}+\sum_{k \in\left\{d_{k} \in D_{2}\right\}} P_{k} W_{2}+\sum_{k \in\left\{d_{j} \in D_{j}\right\}} P_{k} W_{3}} \text { eq. } 3
$$

where $S_{j}$ is the number of hospital beds and the $P_{k}$ terms correspond to the population falling within each of the three travel time subzones $\left(D_{1}, D_{2}, D_{3}\right)$.

In the second step, for each population location, the supply to demand ratios for all hospitals were summed, weighted by their location in the three subzones:

$$
W A_{i}^{F}=\sum_{j \in\left\{d_{i} \in D_{r}\right\}} R_{j} W_{r}=\sum_{j \in\left\{d_{i} \in D_{1}\right\}} R_{j} W_{1}+\sum_{j \in\left\{d_{j} \in D_{2}\right\}} R_{j} W_{2}+\sum_{j \in\left\{d_{i j} \in D_{3}\right\}} R_{j} W_{3} \text { eq. } 4
$$

where $W A_{l}^{F}$ represents the accessibility of hospital beds for the population at location $i$.

We examined the effectiveness of the traditional method in identifying under-served areas by comparing its results with the E2SFCA results. Since the lowest level of administrative data available is the county level, the E2SFCA-based $W A_{i}^{F}$ values were aggregated to the county level to make the outputs of the two methods comparable. The aggregated value $\left(A_{c}^{F}\right)$ was calculated as the population weighted mean of the subpopulation accessibility values $\left(W A_{l}^{F}\right)$ within each county:

$$
A_{c}^{F}=\frac{\sum_{i=1}^{n} P_{i} W A_{i}^{F}}{\sum_{i=1}^{n} P_{i}}
$$

where $c$ denotes the county, and $n$ is the number of locations $i$ within county $c$. 
Lastly, we calculated relative accessibility indices for both the traditional and E2SFCA method results:

$$
R A_{c}^{T}=\frac{A_{c}^{T}-\overline{A_{c}^{T}}}{\overline{A_{c}^{T}}}
$$

and

$$
R A_{c}^{F}=\frac{A_{c}^{F}-\overline{A_{c}^{F}}}{\overline{A_{c}^{F}}}
$$

where $\underline{A_{c}{ }^{T}}$ and $\underline{A_{c}{ }^{F}}$ are the ratios of population to hospital services within county $c$. and are the arithmetic means of $A_{c}{ }^{T}$ and $A_{c}{ }^{F}$ at province level, respectively. The numerators represent the accessibility differences between the specific county and the provincial average, while dividing by the provincial mean standardises the indices' scales.

The mapping and spatial analysis were performed using ArcGIS 9.3 (ESRI, 2009), and the statistical analysis was performed using Stata 12 (StataCorp 12, 2011).

\section{Results}

The results of the shortest path analysis can be seen in Figure 4. As expected, accessibility was found to be highly uneven throughout the province, with the central eastern part having the highest accessibility with most residents living within 10 minutes or less of the nearest hospital.

The average travel time to the nearest hospital for residents of Sichuan Province was found to be 48.4 minutes, $60 \%$ longer than the 30-minute standard used for hospital care (Bosanac et al., 1976; Delamater et al., 2012). Using this approach, we found that 31.8 million people (39.4\% of Sichuan's population) should be able to access the nearest hospital within 30 minutes (Table 2). More detailed results of the shortest path analysis show that 29.8 million (36.8\%), 13.9 million (17.2\%) and 5.3 million (6.6\%) can access the nearest hospital within 30-60 minutes, 1-2 hours, and more than 2 hours, respectively.

The spatial accessibility results provided by the E2SFCA for hospital beds, health professionals and doctors are displayed in Figures 5-7, respectively.

As measured by the E2SFCA, the geographic patterns of spatial accessibility to the three hospital care indicators appeared to be similar throughout the province. However, when comparing the E2SFCA results with the county-based ratio method, a notable difference with regard to

\begin{tabular}{|c|c|c|c|c|c|c|c|c|}
\hline \multirow{2}{*}{$\begin{array}{l}\text { Average travel } \\
\text { time (min) }\end{array}$} & \multicolumn{2}{|c|}{$<30$ min travel } & \multicolumn{2}{|c|}{$30-60 \mathrm{~min}$ travel } & \multicolumn{2}{|c|}{$60-120 \mathrm{~min}$ travel } & \multicolumn{2}{|c|}{$>120$ min travel } \\
\hline & $\begin{array}{l}\text { Number } \\
\text { of persons } \\
\text { (million) }\end{array}$ & $\begin{array}{c}\text { Share of } \\
\text { population } \\
\text { (\%) }\end{array}$ & $\begin{array}{l}\text { Number } \\
\text { of persons } \\
\text { (million) }\end{array}$ & $\begin{array}{c}\text { Share of } \\
\text { population } \\
(\%)\end{array}$ & $\begin{array}{l}\text { Number } \\
\text { of persons } \\
\text { (million) }\end{array}$ & $\begin{array}{l}\text { Share of } \\
\text { population } \\
(\%)\end{array}$ & $\begin{array}{l}\text { Number } \\
\text { of persons } \\
\text { (million) }\end{array}$ & $\begin{array}{c}\text { Share of } \\
\text { population } \\
(\%)\end{array}$ \\
\hline$\underline{48.4}$ & 31.8 & 39.4 & 29.8 & 36.8 & 13.9 & 17.2 & 5.3 & 6.6 \\
\hline
\end{tabular}

Table 2. Average and estimated travel time to the closest hospital for populations at different distances.

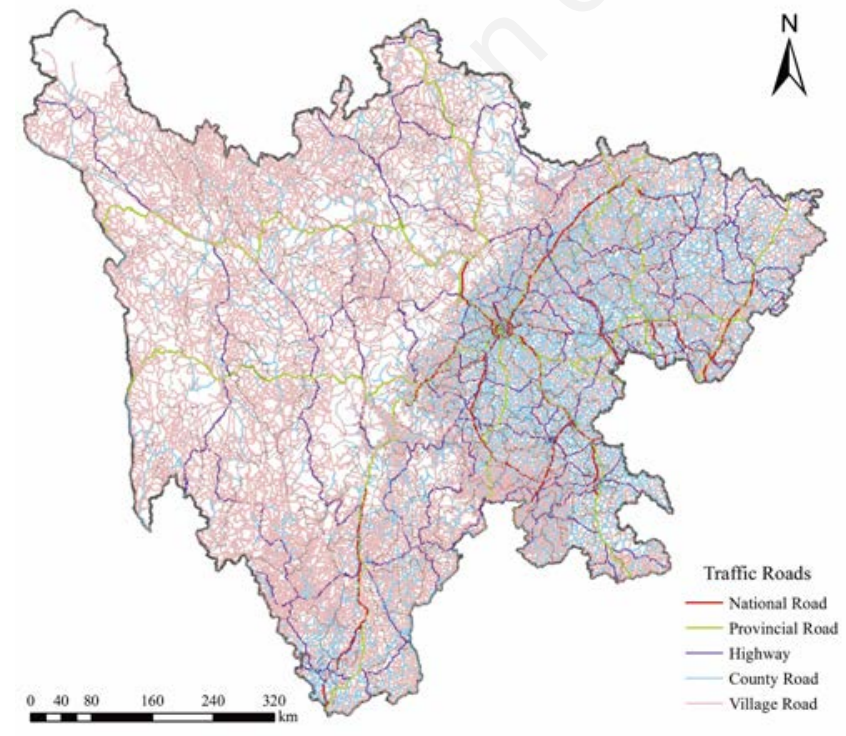

Figure 3. Traffic network as of 2012 in Sichuan Province, China.

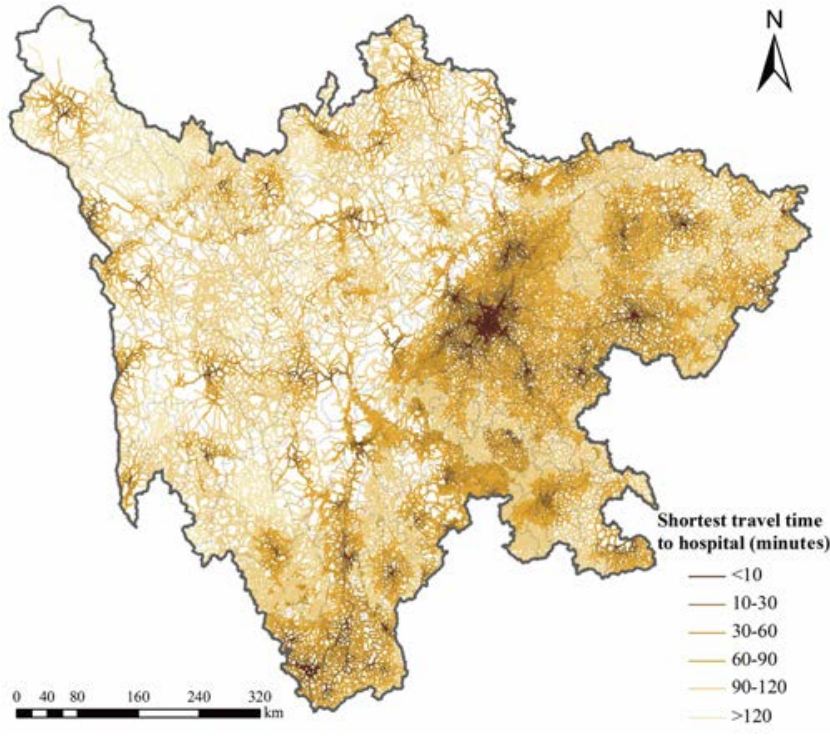

Figure 4. Shortest travel time to a hospital in Sichuan Province, China. 

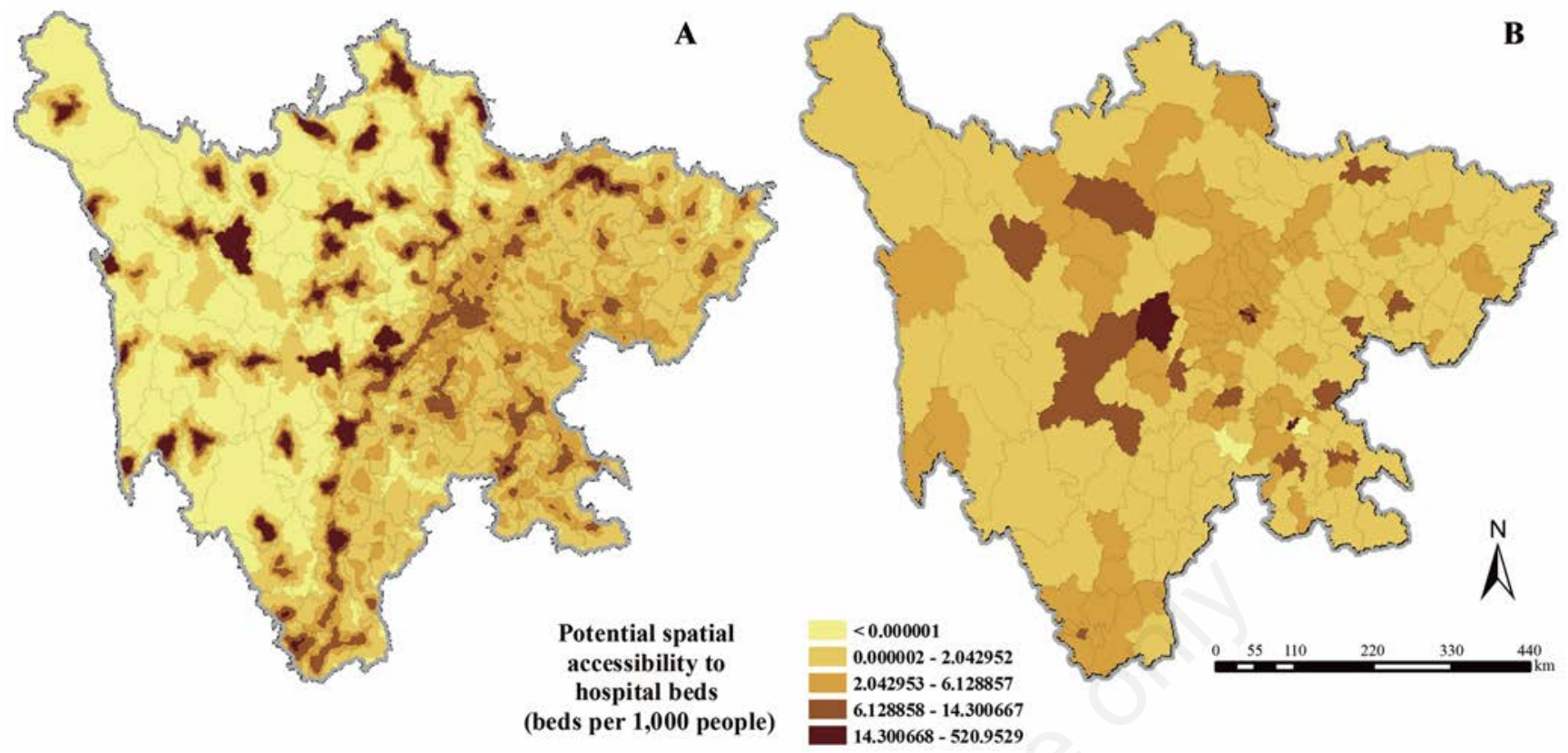

Figure 5. Spatial accessibility to hospital beds: A) enhanced two-step floating catchment area; B) county based ratio.
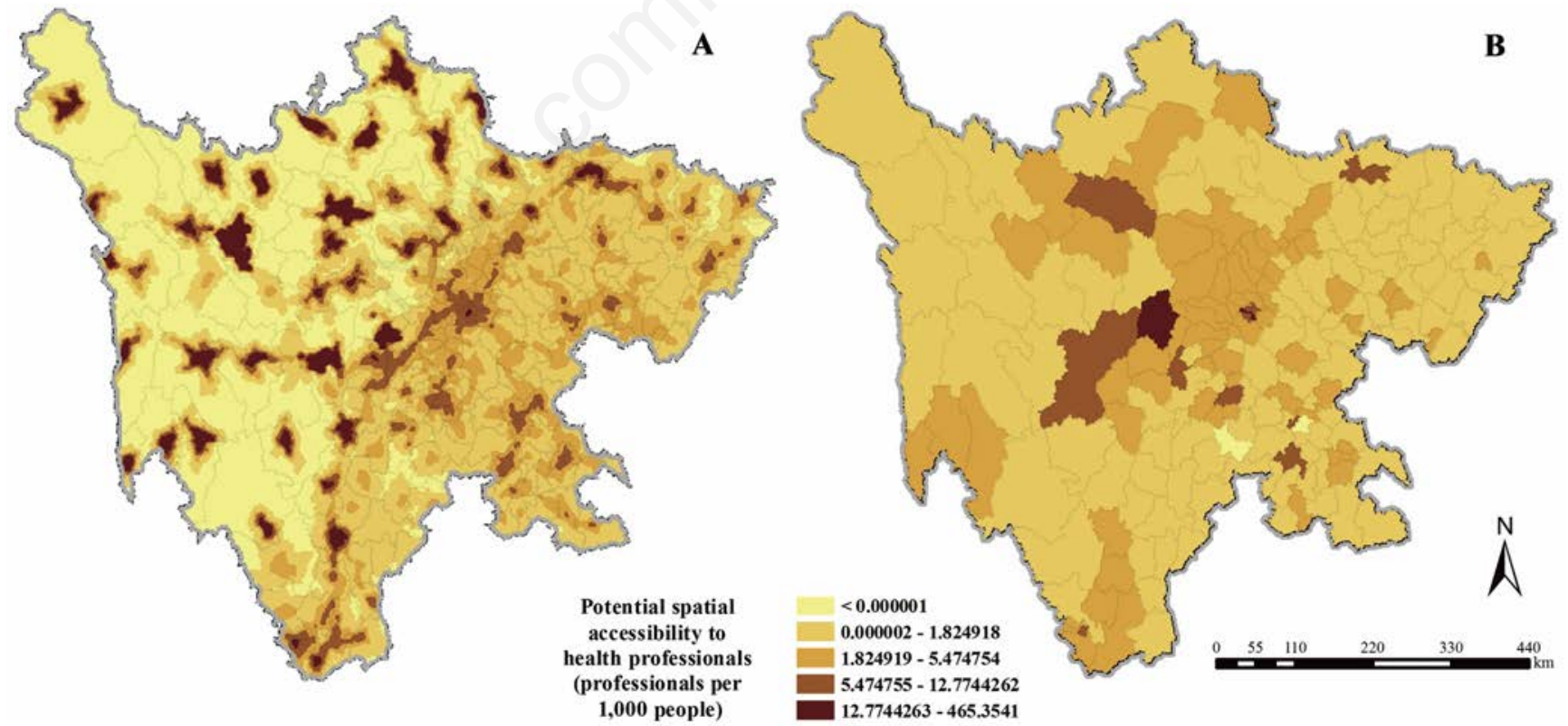

Figure 6. Spatial accessibility to any hospital staff: A) enhanced two-step floating catchment area; B) county based ratio. 
variation within the counties was noted. Importantly, the E2SFCA results show both under-served and high-accessibility areas within the same county.

The E2SFCA results also differ from the geographical distribution of hospitals (Figure 2) and the shortest path analysis results (Figure 4). The biggest differences were found in the less populated western counties, where areas characterised by high spatial accessibility was identified by the E2SFCA approach. The differences in these results underscore the importance of integrating supply, potential demand and distance when evaluating spatial accessibility.

The E2SFCA and the county-based ratio results were compared by calculating the correlation coefficient and via paired t-tests. Table 3 presents the results. Pearson's correlation coefficients for the three indicators of hospital supply capacity were all slightly above 0.5 , indicating a moderate, positive relationship between the results, while the paired t-tests showed that the mean values were not significantly different $(\mathrm{P}<0.05)$ for any of the three indicators.

Figure 8 shows the results of the relative accessibility analysis. Maps in the left column show the E2SFCA results, with the county-based ratios displayed on the right. In general, the results of the two methods appear similar at the county level, which is consistent with the correlation and paired t-test results.

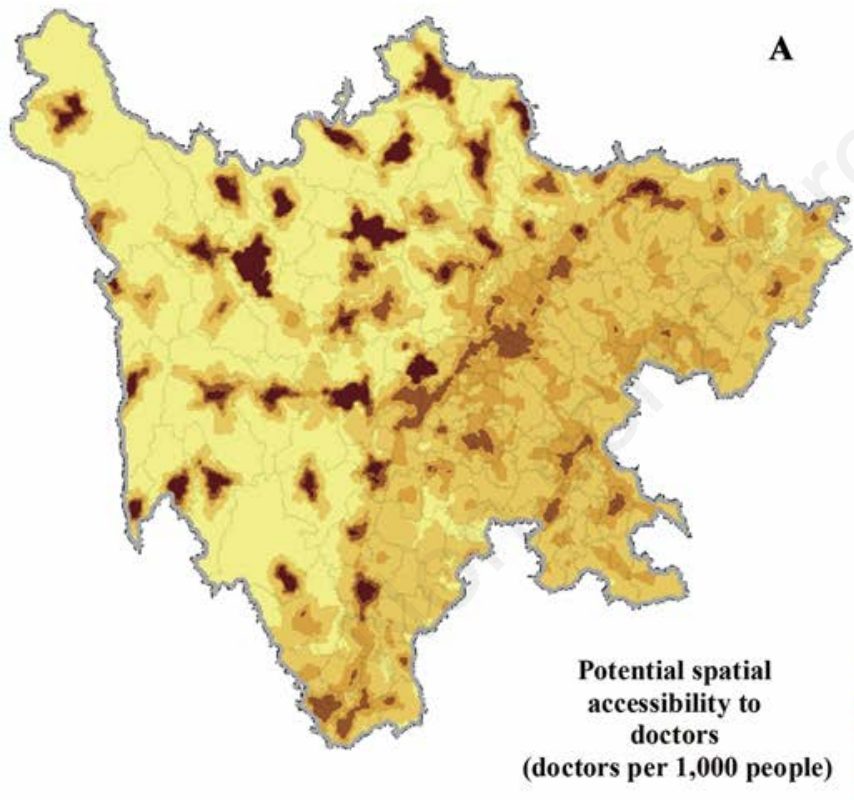

\section{Discussion}

For both methods, counties were categorised into two groups based on whether they were above or below the provincial average and compared. Although a large majority of the counties were identified as being in the same group by both methods, 32 (17.7\% of all counties), $31(17.1 \%)$ and $25(13.8 \%)$ counties were inconsistently classified for hospital beds, health professionals and hospital practitioners, respectively (Table 4). Given the previously discussed advantages of the E2SFCA method, the inconsistencies imply that the traditional approach has the potential to misrepresent relative spatial accessibility for a nontrivial number of counties in Sichuan Province.

This research aimed to: i) apply a more comprehensive GIS-based method to measure spatial accessibility for a healthcare system serving a large area and population, in which advanced spatial analytic approaches have been rarely applied for health planning, and ii) assess the effectiveness of the conventional method (county-based per population ratios) for identifying underserved regions by comparing the results to the E2SFCA method results.

Of the 80.76 million residents of Sichuan province, we found that there are 5.5 million residents (6.6\% of total population) who live more

Figure 7. Spatial accessibility to hospital-based doctors: A) enhanced two-step floating catchment area; B) county based ratio.

Table 3. Comparison of accessibility by enhanced two-step floating catchment area and per capita ratio across counties based on correlation coefficient and paired t test.

\begin{tabular}{lccc}
\hline Indicator & Correlation coefficient & Paired t test & P \\
Accessibility to hospital beds & & -1.683 & 0.094 \\
Accessibility to hospital health professionals & 0.512 & -1.362 & 0.175 \\
\hline Accessibility to hospital practitioners & 0.556 & -1.140 & 0.256 \\
\hline
\end{tabular}


than 2 hours travel time from the nearest hospital. Given the golden hour - i.e. the Golden Hour refers to the time period following a traumatic injury during which there is the highest probability that medical treatment will prevent death - as a benchmark of risk due to limited geographic accessibility, this information suggests a large number of people are potentially at high risk throughout the region.

Spatial accessibility to hospital care varies dramatically throughout the province. Generally, spatial accessibility is higher in the more heav-
Table 4. Comparison of relative accessibility by enhanced twostep floating catchment area and per capita ratio within county.

\begin{tabular}{llc} 
& \multicolumn{2}{c}{ Inconsistencies } \\
Indicator & n & $\%$ \\
Relative accessibility to hospital beds & 32 & 17.68 \\
Relative accessibility to hospital health professionals & 31 & 17.13 \\
\hline Relative accessibility to hospital practitioners & 25 & 13.81 \\
\hline
\end{tabular}
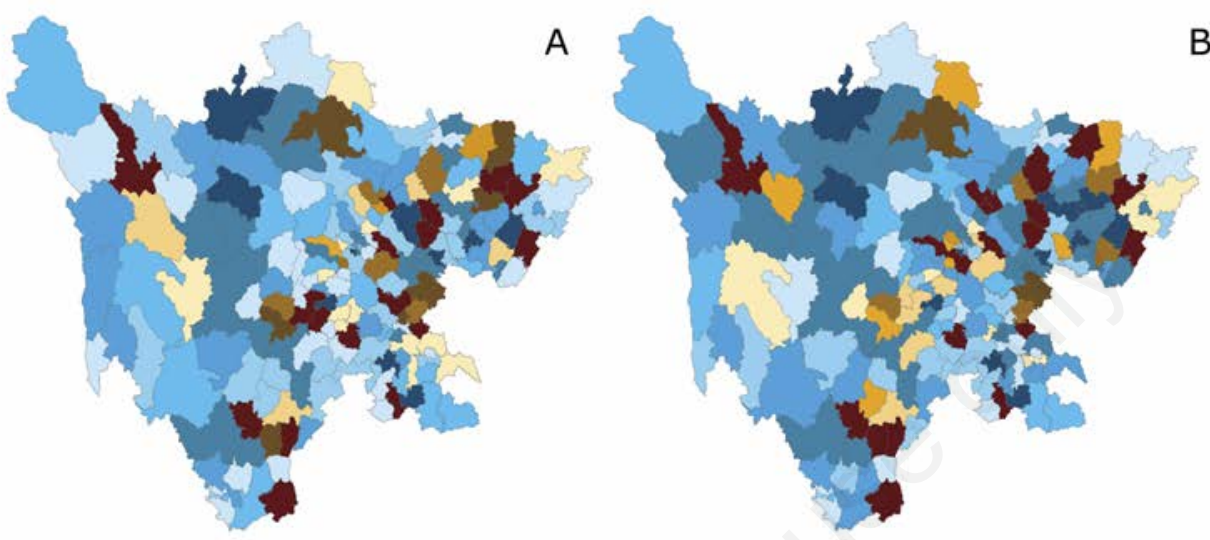

B
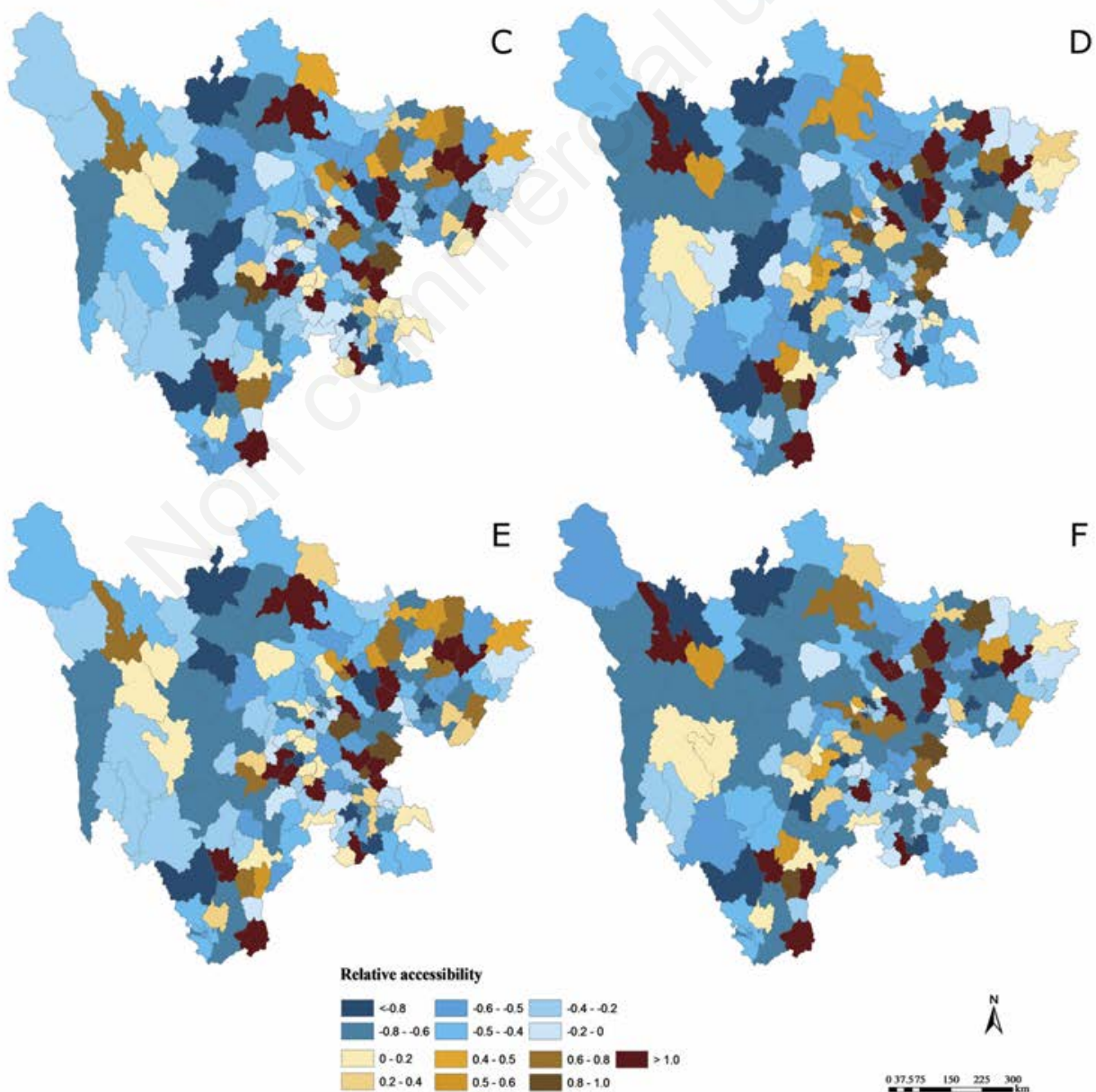

$\mathrm{F}$

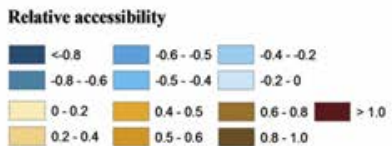

$\AA^{N}$

Figure 8. Comparison of relative accessibility by the enhanced two-step floating catchment area method (left column) and by the per capita ratio at the county level (right column): A, B) beds; C, D) health professionals; E, F) doctors. 
ily populated regions in the eastern portion of the country. However, the variations within the counties were also observed. Notably, the E2SFCA identifies both underserved and high accessibility areas within a single county. Further, the two methods had a roughly $14-18 \%$ difference in classifying counties as above or below the provincial average. These results, in combination with the known limitations of the traditional approach, highlight the benefits provided by implementing the E2SFCA method to assess province-level spatial accessibility.

After the new round of healthcare reforms launched in 2009, China's central and local governments are making large investments in the healthcare sector, including constructing new hospitals and proving support for existing hospitals' development. Determining how to allocate these resources to address equity, while also maximising efficiency remains a challenge. Evidence-based planning is essential for policy makers and our results show that GIS-based approaches, such as the shortest path analysis and the E2SFCA method have the potential to provide important information in these efforts. Further, the E2SFCA could also be extended to examine the spatial distribution of additional healthcare resources including disease-specific healthcare resources or medical equipment.

\section{Conclusions}

Ultimately, we hope that the results presented in this work provide useful information for future healthcare planning and public health policy endeavours. We recommend further integration of spatial analysis for related research in China and suggest that policy makers take note of the shortcomings we have identified regarding per population ratios of healthcare services (within administrative boundaries) and incorporate more comprehensive measures in their efforts.

\section{References}

Aday LA, Andersen R, 1974. A framework for the study of access to medical care. Health Serv Res 9:208.

Andersen RM, Rice TH, Kominski GF, 2011. Changing the US health care system: key issues in health services policy and management. John Wiley \& Sons, New York, NY, USA.

Berke EM, Shi X, 2009. Computing travel time when the exact address is unknown: a comparison of point and polygon ZIP code approximation methods. Int J Health Geogr 8:23.

Bosanac EM, Parkinson RC, Hall DS, 1976. Geographic access to hospital care: a 30-minute travel time standard. Med Care 14:616-23.

Communist Party of China, 2009. The principles of the health care reform. Central Committee and State Council, Beijing, China.

Cromley EK, McLafferty SL, 2011. GIS and public health. 2nd ed. Guilford Publ., New York, NY, USA.

Delamater PL, 2013. Spatial accessibility in suboptimally configured health care systems: a modified two-step floating catchment area (M2SFCA) metric. Health Place 24:30-43.

Delamater PL, Messina JP, Shortridge AM, Grady SC, 2012. Measuring geographic access to health care: raster and network-based methods. Int J Health Geogr 11:15.

Development and Reform Commission of China, 2012. Health care reform in the past three years achieves the goals and benefits millions of people. Development and Reform Commission of China,
Beijing, China.

Dewulf B, Neutens T, De Weerdt Y, Van de Weghe N, 2013. Accessibility to primary health care in Belgium: an evaluation of policies awarding financial assistance in shortage areas. BMC Fam Pract 14:1-13.

ESRI, 2009. Release 9.3. Environmental Systems Research Institute Redlands, CA, USA. Available from: http://www.esri.com/ software/arcgis

Florin J, Gesler WM, Savitz LA, Fondren LK, 1994. Regionalization of health care, Geographic Methods for Health Services Research. University Press of America, Lanham, MD, USA.

Guagliardo MF, 2004. Spatial accessibility of primary care: concepts, methods and challenges. Int J Health Geogr 3:3.

Horev T, Pesis-Katz I, Mukamel DB, 2004. Trends in geographic disparities in allocation of health care resources in the US. Health Policy 68:223-32.

Joseph AE, Bantock PR, 1982. Measuring potential physical accessibility to general practitioners in rural areas: a method and case study. Soc Sci Med 16:85-90.

Joseph AE, Phillips DR, 1984. Accessibility and utilization: geographical perspectives on health care delivery. Harper and Row, New York, NY, USA.

Khan AA, 1992. An integrated approach to measuring potential spatial access to health care services. Socio Econ Plan Sci 26:275-87.

Kivell P, Turton B, Dawson B, 1990. Neighbourhoods for health service administration. Soc Sci Med 30:701-11.

Kwan MP, 1998. Space time and integral measures of individual accessibility: a comparative analysis using a point based framework. Geogr Anal 30:191-216.

Laditka JN, Laditka SB, Probst JC, 2009. Health care access in rural areas: evidence that hospitalization for ambulatory care-sensitive conditions in the United States may increase with the level of rurality. Health Place 15:761-70.

Langford M, Fry R, Higgs G, 2012. Measuring transit system accessibility using a modified two-step floating catchment technique. Int $\mathbf{J}$ Geogr Inf Sci 26:193-214.

Luo W, Qi Y, 2009. An enhanced two-step floating catchment area (E2SFCA) method for measuring spatial accessibility to primary care physicians. Health Place 15:1100-7.

Luo W, Wang F, 2003. Measures of spatial accessibility to health care in a GIS environment: synthesis and a case study in the Chicago region. Environ Plann B 30:865-84.

Makuc DM, Haglund B, Ingram DD, Kleinman JC, Feldman JJ, 1991. The use of health service areas for measuring provider availability. J Rural Health 7:347-56.

McGrail MR, 2012. Spatial accessibility of primary health care utilising the two step floating catchment area method: an assessment of recent improvements. Int J Health Geogr 11:50.

McGrail MR, Humphreys JS, 2009. Measuring spatial accessibility to primary care in rural areas: improving the effectiveness of the twostep floating catchment area method. Appl Geogr 29:533-41.

National Bureau of Statistics of China, 2013. China statistical yearbook 2012. National Bureau of Statistics of China, Beijing, China.

Penchansky R, Thomas JW, 1981. The concept of access: definition and relationship to consumer satisfaction. Med Care 19:127-40.

Radke J, Mu L, 2000. Spatial decompositions, modeling and mapping service regions to predict access to social programs. Lect Notes Comput Sc 6:105-12.

Ranga V, Panda P, 2014. Spatial access to inpatient health care in northern rural India. Geospat Health 8:545-56.

StataCorp 12, 2011. Stata survey data reference manual, release 12. StataCorp, College Station, TX, USA. 
State Council, 2010. Urban resident basic medical insurance household survey. State Council, Beijing, China.

Wan N, Zou B, Sternberg T, 2012. A three-step floating catchment area method for analyzing spatial access to health services. Int J Geogr Inf Sci 26:1073-89.

Wang L, 2007. Immigration, ethnicity, and accessibility to culturally diverse family physicians. Health Place 13:656-71.
Weibull JW, 1976. An axiomatic approach to the measurement of accessibility. Reg Sci Urban Econ 6:357-79.

Wing P, Reynolds C, 1988. The availability of physician services: a geographic analysis. Health Serv Res 23:649.

World Health Organization, 2000. The world health report 2000: health systems: improving performance. World Health Organization, Geneva, Switzerland. 